\title{
Genomics of drug resistance:
}

\section{moving ahead}

\begin{abstract}
Sanjay Sisodiya
Department of Clinical and Epilepsy, UCL Institute of Neurology, Queen Square, London WC1N 3BG, UK
\end{abstract}

Key words: Drug resistance, anti-epileptic drug, genomics, transporter, p-glycoprotein

Published online April 7, 2010

Drug resistance is an important clinical problem. Significant efforts have been made recently to improve understanding. Most recently, a Commission of the International League against Epilepsy has produced a document on the definition of drug resistance [1]. This is an important step forward. It must be recognised, however, that the definition of drug resistance may need to be adjusted according to the needs of a particular clinical or research question. Overall, about one in three patients who have epilepsy do not respond to currently available anti-epileptic drugs. Not only is this a significant burden on healthcare systems, but it is also associated with an in- creased risk of morbidity and premature mortality for patients, as well as a broad range of psycho-social consequences. One can estimate that in the United Kingdom alone, for example, the cost of the first failed anti-epileptic drug is of the order of $£ 20$ to $£ 30$ million per year. There are of course costs well beyond monetary values. Observational studies have demonstrated that some patients who have not responded to several anti-epileptic drugs in the past, and might therefore be expected to continue to have seizures despite trials of further anti-epileptic drugs [2], might in fact occasionally respond to a new anti-epileptic drug $[3,4]$. 
Genomic investigation in drug-resistant epilepsy has a number of roles. Firstly, it may help us better understand the mechanisms of drug resistance. Secondly, it may allow prediction of the drug resistant state. This, however, is of limited use as we can offer little to most patients who might be predicted to have drug-resistant epilepsy, and unless such a prediction were absolutely certain, anti-epileptic drugs are still the most likely treatment option. It may also be that genomic investigation of drug resistance might identify the best available drug for an individual patient, and this certainly is an important short-term goal.

A broader philosophical question is whether the set of genomic variants that might contribute to drug resistance are equivalent to, or at least partly overlap, the set of genetic variants that confer genetic susceptibility to epilepsy and the genetic contribution to variation in drug response. In some cases, it appears as if the same variant might in fact influence disease susceptibility, drug response and drug resistance. The best example to date is Dravet's Syndrome caused by mutations in the SCN1A gene [5]. Alternatively, it may be that separate non-overlapping sets of variants influence these three aspects of any epilepsy. It is likely that in any individual, multiple factors are operating, and different sets of factors may be active in different patients. Different genetic variants may contribute to these three different aspects of an epilepsy in an individual patient. The underlying belief motivating genomic studies of drug resistance is that rational therapy for pharmacoresistance will emerge from understanding causes of pharmacoresistance.

It is always important in clinical practice or research to be certain that patients who are considered to have drug-resistant epilepsy do truly demonstrate drug resistance. It is important to be clear that the resistance is not apparently, for example, due to non-compliance. When this has been clarified, one also needs to know whether drug resistance is to a single drug or multiple drugs, drugs that operate in different ways and have different pharmacokinetics. It is also important to appreciate that drug resistance may be a dynamic phenomenon. The patient who has been seizurefree for a year today, may have seizure recurrence tomorrow and then fail to be controlled despite the use of several anti-epileptic drugs over a period of years [6]. This phenomenon complicates any evaluation of drug resistance.

For some years, the transporter hypothesis has held considerable sway in the study of drug resistance [7]. The hypothesis when initially aired at least had biological plausibility. Immunohistochemical studies demonstrated the presence of multidrug transporters, at least at a low resolution, in human brain tissue from patients with drug-resistant epilepsy. Early studies suggested that the archetypal multidrug transporter, P-glycoprotein, was capable of transporting anti-epileptic drugs. The difficulty in evaluating the function of Pglycoprotein in human brain in vivo led to a search for alternative means of determining its function. This led to one of the first pharmacogenetic studies of drug resistance in epilepsy [8]. In this study, one particular polymorphism (C3435T) in the ABCB1 gene en- 
coding P-glycoprotein was studied in a cohort of patients of mainly Western European origin, who had either drug-resistant or drugsensitive epilepsy. At that stage, the CC genotype was known to be associated in Western European populations with an increased amount and activity of Pglycoprotein, at least as assessed in intestine. An association was identified between the CC genotype and the clinical phenotype of drug resistance.

However, the ensuing years have led to much uncertainty about the role for this particular polymorphism $[9,10]$. A large number of attempted replications have been undertaken. Few of these have been true replications of the original finding. The one true replication of reasonable size did not replicate the original finding [11]. Most of the replication attempts have studied patients with different phenotypes, with different definitions for drug resistance or drug sensitivity. At a genetic level, the role for this particular variant in $A B C B 1$ is therefore still unclear, though it would seem that it is unlikely to have a major role in most patients with epilepsy.

Nevertheless, there is evidence that this polymorphism can influence P-glycoprotein activity or quantity. A study in human liver tissue suggests that there is allelic imbalance, and that the $\mathrm{C}$-allele produces an mRNA that seems to be more stable [12]. Therefore even though this polymorphism is synonymous, it may still have an influence on P-glycoprotein. In addition other studies have shown that different polymorphisms in the $A B C B 1$ gene can influence P-glycoprotein activity at least in model systems in vitro, with an effect particularly noted for a stress on gene expression, possibly related to codon usage bias [13]. Therefore it may still be the case that the C3435T polymorphism does have an influence on P-glycoprotein activity and function, and therefore conceivably on drug resistance in epilepsy. An important idea emerging from these in vitro studies is that stress on gene expression is an important component of analysis. Therefore comparison of patients with new onset epilepsy with those with chronic epilepsy may not be appropriate. It also may not be appropriate to compare any patient with drug-resistant epilepsy with any patient with drug-sensitive epilepsy; it may be necessary to take detailed account of the drugs to which patients have been exposed, and on which they are being treated at the time of the study.

The transporter hypothesis for drug resistance in epilepsy therefore remains unproven. More information is needed before multidrug transporters can be accepted as having a role to play in drug resistance in epilepsy. A framework is required for the evaluation of the candidacy of any particular mechanism in drug resistance [7]. These have been suggested for multidrug transporters already.

For example, one might consider it necessary to demonstrate that multidrug transporters are located in the right position orientation and quantity in human epileptogenic brain tissue in comparison to normal tissue, that multidrug transporters should be able to transport anti-epileptic drugs of a variety of different types, and that inhibition of the activity of transporters should lead to an im- 
provement in the control of drug-resistant epilepsy.

One key aspect of the transporter hypothesis implicit in its formulation but rarely considered explicitly, is that there should be more P-glycoprotein activity (total activity or activity per molecule) in the epileptogenic region in comparison to non-epileptogenic regions even within a single brain. This of course is difficult to test using surgically-resected brain tissue, as in general tissue is only resected from epileptogenic regions and the surrounding area. In the future, we could hope that in vivo imaging of multidrug transporter activity may help us address this question. Such studies are ongoing. An alternative approach is to study whole post-mortem brain from patients who had epilepsy and subsequently died. One could then compare patterns of expression of P-glycoprotein within the epileptogenic region compared with putatively nonepileptogenic regions, to see whether this implicit assumption of the transporter hypothesis can be upheld. Preliminary data suggests that this is indeed the case. Further studies are still required, for example to examine the polarity of activity of P-glycoprotein, to consider redundancy of functional activity between overlapping transporters, and to consider their relative contribution to drug resistance.

Whilst the transporter hypothesis to some at least has been seductive, it is important to note that there are other possible mechanisms to mediate drug resistance. One, for example, is to consider a more general breakdown of the blood-brain barrier beyond multidrug transporter activity alone. Some published evi- dence suggests that there is increased permeation of albumin, an epileptogenic molecule, into epileptogenic brain tissue and that this might contribute to epileptogenesis [14]. Other studies in model systems show an important role for leukocyte infiltration [15], again underlining the importance of the bloodbrain barrier and its disruption in acute and chronic epilepsy.

Very elegant studies of an animal model of Dravet's syndrome nicely demonstrate that there can be very cell-specific and spatiallyrestricted alteration in ion channel activity, that may be genetically mediated, that can lead to an imbalance between excitation and inhibition and contribute to epileptogenesis [16] as well as drug resistance. Whether such mechanisms apply to the Dravet's syndrome in humans has yet to be proven, but the animal model data are consistent with observational studies in humans showing that certain antiepileptic drugs that further inhibit sodium channel activity, such a lamotrigine, can aggravate seizure activity in Dravet's syndrome. This is an exciting area that promises to reveal much more about general processes of epileptogenesis in humans.

With the limited success to date of possible mechanisms of drug resistance in humans, it is now time to take a step back and consider a broader perspective on mechanisms. It is important to consider the biology of the epilepsy of anti-epileptic drugs and drug resistance.

The technology, methodology, and cohorts now exist to undertake large scale, broader perspective studies of drug resistance. In particular, genome-wide association studies of 
drug response in epilepsy may well hold potential for improving our understanding of drug resistance. The technology and methodology of genome-wide association studies is now mature. There are a large number of studies that have been published both for disease susceptibility and normal human trait variation, such as height and weight. In comparison, there are still relatively few pharmacogenomic studies. The studies that have been undertaken underline the importance of clearly defining the phenotype, of accurate quality controlled genotyping, of the study of large, appropriately-chosen cohorts, and of careful analysis replication and follow-up. Whilst the processes are complex, time consuming, and expensive, they are certainly possible.

There are a number of large cohorts of patients with epilepsy that have been collected across the world. Many of these have been phenotyped in detail. Genome-wide association studies of drug response are underway. It is to be hoped that these studies will reveal possible mechanisms. In comparison to disease susceptibility studies, where genomewide association studies have revealed a large "missing heritability" gap [17], it is to be hoped that the lack of evolutionary pressure that humans have been exposed to for manmade drugs will mean that variants of significant effect are still common in the population and therefore still detectable using genomewide association strategies. These are exciting times in the genomic study of drug resistance.

\section{References}

[1] Kwan P, Arzimanoglou A, Berg AT, Brodie MJ, Allen Hauser W, Mathern G, Moshé SL, Perucca E, Wiebe S, French J. Definition of drug resistant epilepsy: Consensus proposal by the ad hoc Task Force of the ILAE Commission on Therapeutic Strategies. Epilepsia 2009; Nov 3. [Epub ahead of print]

[2] Kwan P, Brodie MJ. Effectiveness of first antiepileptic drug. Epilepsia 2001; 42, 12551260.

[3] Luciano AL, Shorvon SD. Results of treatment changes in patients with apparently drug-resistant chronic epilepsy. Ann Neurol 2007; 62: 375-381.

[4] Callaghan BC, Anand K, Hesdorffer D, Hauser WA, French JA. Likelihood of seizure remission in an adult population with refractory epilepsy. Ann Neurol 2007; 62: 382-389.

[5] Mullen SA, Scheffer IE. Translational research in epilepsy genetics: sodium channels in man to interneuronopathy in mouse. Arch Neurol 2009; 66: 21-26.

[6] Berg AT. The natural history of mesial temporal lobe epilepsy. Curr Opin Neurol 2008; 21: 173-178.

[7] Sisodiya SM. Mechanisms of antiepileptic drug resistance. Curr Opin Neurol 2003; 16 : 197-201.

[8] Siddiqui A, Kerb R, Weale ME, Brinkmann U, Smith A, Goldstein DB, Wood NW, Sisodiya SM. Association of multidrug resistance in epilepsy with a polymorphism in the drugtransporter gene ABCB1. N Engl J Med 2003; 348: 1442-1448.

[9] Bournissen FG, Moretti ME, Juurlink DJ, Koren G, Walker M, Finkelstein Y. Polymorphism of the MDR1/ABCB1 C3435T drug-transporter and resistance to anticonvulsant drugs: A meta-analysis Epilepsia 
2009; 50: 898-903.

[10] Kasperaviciūte D, Sisodiya SM. Epilepsy pharmacogenetics. Pharmacogenomics 2009; 10: 817-836.

[11] Tan NC, Heron SE, Scheffer IE, Pelekanos JT, McMahon JM, Vears DF, Mulley JC, Berkovic SF. Failure to confirm association of a polymorphism in ABCB1 with multidrug-resistant epilepsy. Neurology 2004; 63: 1090-1092.

[12] Wang D, Johnson AD, Papp AC, Kroetz DL, Sadée W. Multidrug resistance polypeptide 1 (MDR1, ABCB1) variant 3435C $>\mathrm{T}$ affects mRNA stability. Pharmacogenet Genomics 2005; 15: 693-704.

[13] Kimchi-Sarfaty C, Oh JM, Kim IW, Sauna ZE, Calcagno AM, Ambudkar SV, Gottesman MM. A "silent" polymorphism in the MDR1 gene changes substrate specificity. Science 2007; 315: 525-528. Erratum in: Science 2007; 318: 1382-1383.

[14] van Vliet EA, da Costa Araújo S, Redeker S, van Schaik R, Aronica E, Gorter JA. Bloodbrain barrier leakage may lead to progression of temporal lobe epilepsy. Brain 2007; 130(Pt 2): 521-534.

[15] Fabene PF, Navarro Mora G, Martinello M, Rossi B, Merigo F, Ottoboni L, Bach S, Angiari S, Benati D, Chakir A, Zanetti L, Schio F, Osculati A, Marzola P, Nicolato E, Homeister JW, Xia L, Lowe JB, McEver RP, Osculati F, Sbarbati A, Butcher EC, Constantin G. A role for leukocyte-endothelial adhesion mechanisms in epilepsy. Nat Med 2008; 14: 1377-1383.

[16] Yu FH, Mantegazza M, Westenbroek RE, Robbins CA, Kalume F, Burton KA, Spain WJ, McKnight GS, Scheuer T, Catterall WA. Reduced sodium current in GABAergic interneurons in a mouse model of severe myo- clonic epilepsy in infancy. Nat Neurosci 2006; 9: 1142-1149. Erratum in: Nat Neurosci 2007; 10: 134.

[17] Manolio TA, Collins FS, Cox NJ, Goldstein DB, Hindorff LA, Hunter DJ, McCarthy MI, Ramos EM, Cardon LR, Chakravarti A, Cho JH, Guttmacher AE, Kong A, Kruglyak L, Mardis E, Rotimi CN, Slatkin M, Valle D, Whittemore AS, Boehnke M, Clark AG, Eichler EE, Gibson G, Haines JL, Mackay TF, McCarroll SA, Visscher PM. Finding the missing heritability of complex diseases. Nature 2009; 461: 747-753. 